\title{
FuturICT: Participatory computing to understand and manage our complex world in a more sustainable and resilient way
}

\author{
D. Helbing ${ }^{1, a}$, S. Bishop ${ }^{2}$, R. Conte ${ }^{3}$, P. Lukowicz ${ }^{4}$, and J.B. McCarthy ${ }^{5}$ \\ 1 ETH Zurich, Switzerland \\ 2 Math Department, University College London, UK \\ 3 ISTC-CNR, Rome, Italy \\ 4 DFKI, Kaiserlautern, Germany \\ ${ }^{5}$ University College Cork, Ireland
}

Received 1 August 2012 / Received in final form 9 October 2012 Published online 5 December 2012

\begin{abstract}
We have built particle accelerators to understand the forces that make up our physical world. Yet, we do not understand the principles underlying our strongly connected, techno-socio-economic systems. We have enabled ubiquitous Internet connectivity and instant, global information access. Yet we do not understand how it impacts our behavior and the evolution of society.

To fill the knowledge gaps and keep up with the fast pace at which our world is changing, a Knowledge Accelerator must urgently be created. The financial crisis, international wars, global terror, the spreading of diseases and cyber-crime as well as demographic, technological and environmental change demonstrate that humanity is facing serious challenges. These problems cannot be solved within the traditional paradigms.

Moving our attention from a component-oriented view of the world to an interaction-oriented view will allow us to understand the complex systems we have created and the emergent collective phenomena characterising them. This paradigm shift will enable new solutions to long-standing problems, very much as the shift from a geocentric to a heliocentric worldview has facilitated modern physics and the ability to launch satellites.

The FuturICT flagship project will develop new science and technology to manage our future in a complex, strongly connected world. For this, it will combine the power of information and communication technology (ICT) with knowledge from the social and complexity sciences. ICT will provide the data to boost the social sciences into a new era. Complexity science will shed new light on the emergent phenomena in socially interactive systems, and the social sciences will provide a better understanding of the opportunities and risks of strongly networked systems, in particular future ICT systems. Hence, the envisaged
\end{abstract}

\footnotetext{
${ }^{a}$ e-mail: dhelbing@ethz.ch
} 
FuturICT flagship will create new methods and instruments to tackle the challenges of the $21^{\text {st }}$ century.

FuturICT could indeed become one of the most important scientific endeavours ever, by revealing the principles that make socially interactive systems work well, by inspiring the creation of new platforms to explore our possible futures, and by initiating an era of social and socio-inspired innovations.

\section{Introduction}

Globalisation and technological change have made our world a different place. This has created or intensified a number of serious problems, such as global financial and economic crises, political instabilities and revolutions, the quick spreading of diseases, disruptions of international supply chains, organised crime, international conflict and world-wide terrorism, and increased cyber-risks.

Although the creation of more and more interconnected systems and the pace of innovation in the area of information and communication technologies (ICT) have contributed to the above problems, future ICT can also be key to the solution. It can create unprecedented benefits for our economy and society, based on a whole range of new methods and innovations. For this, ICT must acquire the ability to flexibly adapt to the needs of society. In this way, it can become a stabilising factor fostering transparency, trust, respect for individual rights, and opportunities for participation in social, economic, and political processes. This requires us to establish a new science of multi-level complex, global systems and a co-evolution of ICT with society, by bringing the best knowledge of experts on information and communication systems, complex systems and the social sciences together. The vision of the FuturICT flagship project is to improve our capacity to manage our future, based on a fundamental understanding of the institutional and interaction-based principles that make connected systems work well.

The methods and "Big Data" needed for such a scientific endeavour are now becoming available: it is, therefore, time to make a "Big Science" effort to combine methods and data with theories and models, like in the Human Genome Project. This endeavour should be open, because we need to prevent private monopolies of socioeconomic data, and it should be federated, because joint interdisciplinary efforts are the only way to tackle humanity's global challenges and ensure leadership in socioinspired ICT innovations. The investments into the FuturICT project can benefit citizens and society in many ways: by promoting collective awareness of the impacts of our decisions and actions, by mitigating global problems and systemic risks, and by creating new possibilities to participate in social, economic and political affairs. FuturICT will also build a basis for new spin-offs, business opportunities and jobs. After the age of physical, biological, and technological innovations, FuturICT could lead Europe into the next era - a wave of information-driven social and socio-inspired innovations.

\section{The need for new knowledge in a fundamentally changed world}

Today, neither past knowledge nor established policies seem sufficient anymore to manage our future (see Box 1). This is because technological, social and economic systems are becoming more and more complex, and also mutually interdependent. Recent ICT developments such as ubiquitous connectivity, instant information access and the increased penetration of social networking platforms have had a catalytic effect, speeding up and enforcing such interdependencies and destabilising many established systems. 
BOX 1: Paradigm shift in our understanding of the world

The conventional "medicines" to tackle the problems of our world fail more and more often. But many problems today are due to an out-dated understanding of our world. In fact, our traditional way of thinking is fundamentally wrong, because the world has changed: While its parts still look pretty much the same, we have networked them and made them strongly interdependent. When "self-organisation" sets in, the components' individual properties are no longer characteristic for the system behaviour, but collective behaviour takes over. Group dynamics and mass psychology are two typical examples.

As a consequence of the above, we have to shift our attention from the components of our world to their interactions. In other words, we need a change from an objectoriented to an interaction-oriented view, as it is at the heart of complexity science. This paradigm shift may be of similar importance as the transition from a geocentric to a heliocentric view of the world. It has fundamental implications for the way in which complex techno-socio-economic systems must be managed and, hence, for politics and economics. Focusing on the interactions in a system and the multi-level emergent dynamics resulting from them opens up fundamentally new solutions to long-standing problems (see Box 2).

Strongly connected systems often behave completely different from loosely connected systems and opposed to what our everyday intuition suggests - a situation raising fundamental scientific challenges, but also ethical ones (see Boxes 2 and 3, and Appendix A):

- The dynamics of strongly connected systems with positive feedbacks is faster.

- Extreme events occur more often and can impact the whole system.

- Self-organisation and strong correlations dominate the dynamics of the system.

- The system behaviour is often counter-intuitive, and unwanted feedback or side effects are typical.

- The system behaviour is hard to predict, and planning for the future may not be possible.

- Opportunities for external control are very limited.

- In many cases, not even the most powerful computers can perform an optimisation of the system behaviour in real time, as the number of interacting elements is too large.

- The competition for limited resources implies reduced redundancies in the system and a larger vulnerability to random failures or external shocks.

- The loss of predictability and control lead to an erosion of trust in private and public institutions, which in turn can create social, political, or economic instabilities.

Such strongly coupled systems cannot be managed well in a merely top-down fashion. Rather than controlling the individual elements of a system, it becomes crucial to stimulate a more favourable self-organisation in the whole system by establishing suitable interaction rules (the "rules of the game") (see Table 1). Bottom-up elements often allow for greater flexibility, efficiency, and resilience of the system (see Box 4). 
BOX 2: Some success stories: applications of recent discoveries

Fuelled by recent theoretical progress, the social and complexity sciences have sparked off a number of interesting applications, particularly where both perspectives have been integrated: These applications range from models of self-organisation and segregation, which suggest strategies to reduce crime and conflict, over models of social cooperation, which imply recipes to overcome so-called tragedies of the commons, up to opinion formation models, which are used in prediction markets.

Models of pedestrian dynamics can now help to anticipate and avoid crowd disasters. Models of mobility patterns and traffic breakdowns support congestion avoidance and inform the design of smarter cities. Models of financial systems suggest, how to make them more stable and more resilient to shocks.

Simulations of supply chains facilitate more efficient production systems and provide a better understanding of business cycles. Models of conflict and organised crime will allow one to reduce wars, insurgency, and drug traffic. It has also become possible to perform a real-time measurement and simulation of pandemics, which can be used for scenario-based policy recommendations, e.g. regarding more effective immunisation strategies.

Network theory allows one to understand and improve the resilience of systems. It has furthermore enabled Google's powerful page rank algorithm, and the semantic web as well as trust and recommender systems. In addition, social networking applications and models for the evolution of social groups and communities have mutually inspired each other. Last but not least, models for the emergence of language allow robots to find their own way of communication. All these exciting developments promise a great future and relevance of FuturICT's research.

\section{Understanding and designing future ICT systems as artificial social systems}

Our ICT systems are increasingly suffering from similar problems as those that worry societies: the lack of coordination, instability, inefficient use of resources, conflicts of interest. The recent explosion of cyber-crime and the new notion of cyber-war leave the impression that conventionally operated ICT networks may get out of control (the "flash crash" on May 6, 2010, may serve as an example). This is happening because ICT systems are usually not tested for the systemic interactions of their components.

Yet, ICT systems are complex systems, which are made up of billions of nonlinearly interacting elements (computers, smartphones, software agents etc.). These components communicate with each other and, more and more, they take autonomous decisions based on an internal (or "subjective") representation of the surrounding world and expectations regarding future conditions. This effectively makes them artificial social systems. For example, computer-based automatic trading strategies now perform the majority of transactions in our world's financial system.

The realisation that complex ICT systems cannot be effectively controlled in a top-down manner has led to a large body of research on bio-inspired computing. This research has produced many powerful algorithms (such as genetic or ant algorithms) as well as control paradigms for complex self-organised systems.

However, we still do not know how to effectively build trustworthy and resilient ICT systems on a global scale. From the perspective of the envisaged new science of the co-evolution of ICT and society, FuturICT proposes to go one step beyond bio-inspired 
Table 1. Comparison of weakly connected systems with local or regional interactions (as we tended to have them in the past) with strongly connected, complex systems (as we often have them today). In order to understand systemic risks resulting from the new interdependencies and to develop the ability of integrated risks management, we need to overcome the classical silo thinking and even more than that: We need a new way of thinking, a paradigm shift from focusing on the components of a system to focusing on their non-linear interactions, as studied by complexity science. This paradigm shift may be of similar importance as the one from a geocentric to a heliocentric world view, which has enabled modern physics. In a similar way can the shift from a component- to an interaction-oriented perspective promote a new understanding of our techno-socio-economic-environmental systems, and facilitate new solutions to long-standing problems.

\section{Well controllable systems of the past Complex Systems of today}

Weakly connected or independent system components

Dominated by the system components

Simple system behaviour

Sum of properties of individual components characterizes

system behaviour

Conventional wisdom works well

Well predictable and controllable in top-down fashion
Strongly connected or interdependent system component

Dominated by their interactions

Complex system behaviour

Emergent collective behaviour implies new and often unexpected system behaviour

Counter intuitive behaviour, extreme events are common

Less predictable, management by setting rules for bottom up self-organization

and other current approaches by developing algorithms, control methodologies and architectures that are directly inspired by social processes and structures.

Currently, most information and communication systems are not designed for the collective behaviour that may result from the interaction of their components (the same is also true for socio-economic systems). As we go on connecting these systems more and more densely, this can bring about a lack of robustness (failure tolerance) and a lack of resilience (i.e. a vulnerability to attacks and external shocks), as recent scientific studies reveal. Given the ubiquitous use of ICT systems and our strong dependence on the reliability of these systems, proper design principles for such socially interactive systems must be urgently identified. This ultimately requires fundamental knowledge from the social sciences.

\section{The need for a new science of the co-evolution of ICT and society}

With the emergence of the Internet we have entered an age of instant, global availability of digital information. With the spreading of mobile Internet, instant access has become ubiquitous. Furthermore, social networks help to channel information flows according to social interactions, common interests, joint events, and co-location. 


\section{BOX 3: Ethical information and communication systems}

FuturICT has a strong ethical motivation. In particular, the project will study the impact of information and communication technologies (ICT) on individuals and society, including risks of manipulation. It also wants to promote the development of responsive, responsible and ethical ICT.

Over a 10-year time period, FuturICT likes to provide an open data, simulation, exploration and participatory platform for everyone. This is thought to establish a new public good on which all kinds of services can be built, i.e. it will support both commercial and non-profit activities. To prevent misuse of the platform and enable reliable high-quality services, it will be built on principles of transparency, accountability, reputation, and self-regulation.

FuturICT is not interested in tracking individual behaviour or gathering data on individual actions. Its aim is to understand the macroscopic and statistical interdependencies within the highly complex systems on which we all depend.

The FuturICT project will have a strong research focus on ethical issues, and is committed to informing the public about the use of socio-economic data. For example, FuturICT will promote the development of principles for trustworthy ICT systems and of privacy-respecting data mining technologies that give users more control over their data. It will strongly engage in preventing and counter-acting the misuse of data and the Internet. More broadly, the project will seek public involvement to build and sustain confidence in its values (see Appendix A).

Finally, we consider it as a moral obligation to push the research directions promoted by the FuturICT project forward as quickly as possible. Given the fragility of the financial and economic system, the risks that this may finally impact the stability of our society and promote crime, corruption, violence, riots, and political extremism, or even endanger our democracies and our cultural heritage are not negligible anymore. Quick scientific progress is needed in order to learn how to efficiently stop the on-going cascading effects and downward trends. It is of similar importance to ensure that social and socio-inspired innovations will benefit humanity and not end up in the hands of a few stakeholders, as it partially happened in genetics (especially food production).

We are now at the verge of a situation where any event that happens in the real world is being instantly reflected by some piece of information in the digital world, often delivered automatically to potentially interested people. It has been this "perfect technological storm" that many credit for being a crucial factor for the good as well as the bad in events such as the Arab Spring and the recent London Riots.

While the extent of the actual impact of new ICT on such events is disputed, there can be little doubt that we are witnessing a fundamental change in the way human society and ICT interact. ICT is not anymore just a tool for information exchange and data access. The boundaries between the digital and the physical world are increasingly blurred, as ICT becomes an integral part of the fabric of our society.

Thus, more and more ICT systems are directly coupled to complex social systems, collecting and interpreting information about the real world and autonomously taking actions that influence real-world events. For example, large, unexpected fluctuations in the stock market have been attributed to anomalies in automated trading routines.

Systems are emerging that analyse people's behaviour and help them adjust to a more healthy life style with potentially major pubic health impact. On a more subtle level, the ranking of search results, automatic filtering of news stories, or recommendations of friends on social networks may change opinion trends, consumer behavior or social cohesion within communities. Systems are currently under development that 
will control basic services such as energy production or traffic flows, using complex automated reasoning based on large-scale sensor data.

\section{BOX 4: Resilient self-control and how to make more out of scarce resources}

Many crises result from domino or cascading effects (see Appendix B). Such effects are also responsible for the formation of traffic jams. Indeed, urban traffic flows form a strongly coupled system: traffic flows from different points of origin towards different destinations may significantly influence each other. Classical traffic light control is based on supercomputing centres, which collect flow-rate measurements from many intersections and implement pre-determined control decisions in a top-down fashion. As the decision cannot be optimised in real-time (because there are too many alternative control options), one typically adopts a periodic solution, which is optimal for, say, the typical Monday morning or the time after a soccer match. But the average situation never occurs in reality, since the variability of the flows from one red light phase to another is high. Hence, today's traffic light control is far from optimal. A better approach controls traffic flows aperiodically and bottom-up (or combines top-down and bottom-up elements in a suitable way), letting traffic lights flexibly respond to the actual traffic situation. When measuring not only the outflows from road sections, but also the inflows, a short-term anticipation of groups of vehicles becomes possible. This allows traffic lights to turn green when a vehicle group arrives. It is interesting that a high-level system performance is not reached, when each traffic intersection simply implements the best possible local control (as Adam Smith's principle may suggest). However, everyone profits if neighboring intersections coordinate with each other through short-term flow anticipation: car drivers, users of public transport, bikers, pedestrians, and the environment as well. This example illustrates how complex, highly variable and largely unpredictable systems can be made more efficient and resilient: by a combination of real-time measurements, short-term anticipation, decentralized self-organization ('selfcontrol'), and the interruption of cascading effects (here: the avoidance of spill-over effects). As a result, crises can be prevented or mitigated, and scarce resources (in the above case: space and time) can be used more efficiently.

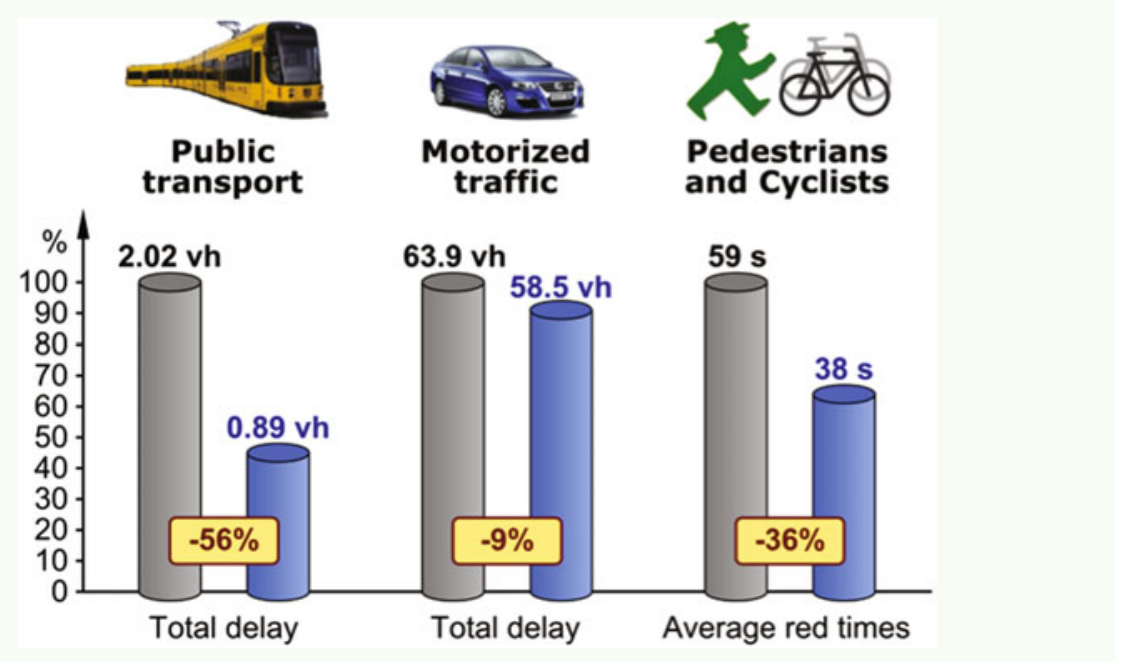

In comparison with a state-of-the-art control (left columns), the flexible self-control (right columns) can reduce the average delay for all modes of transport and is beneficial for our environment as well. 
At the same time, real world events are having more and more impact on the basic functionality of ICT systems. One simple example is the fluctuations in bandwidth requirements related to popular events (e.g. the royal wedding). Another is the cyberattacks that were a direct consequence of disputed political and business decisions (e.g. attacks following attempts to close down the WikiLeaks platform). Furthermore, legal considerations, public opinion, and economic interests have often more influence on the architecture of ICT systems than technical merits of specific solutions. Finally, information on which ICT systems base their decision may be intentionally biased, limited, or censored for political or economic reasons. In summary, society and ICT form a tightly coupled system that needs to be considered as a whole, rather than as two interacting, but separate entities. To this end, FuturICT will develop a new science of the co-evolution of ICT and society, building on an integration of the crucial expertise from the social sciences, computer science, and complexity theory. This new science will enable us to design more resilient ICT systems, to better predict and manage the effects of new technology on our society, and to use ICT technology to support stability, trust, social cohesion, and economic opportunities for the citizens.

\section{The opportunity of creating socially interactive ICT}

Despite the strong coupling between ICT and society, most of today's ICT systems are "blind" to social phenomena. Much research has been devoted to making computers recognise the personal context of an individual, adapt their functionality to individual needs and optimise the interaction between the individual and the system. In contrast, there is very little understanding of the interaction between information technologies and society as a whole. Much of the influence that information technology today has on society is unintended and random. For example, systems that deliver personalised news feeds to users base their decisions on individual preferences, but pay no attention to possible side effects such as a potential radicalisation or fragmentation of public opinion. Automatic trading systems tend to optimise individual or corporate goals, but pay little attention to the consequences of their actions on the market as a whole and potential global crises created by them.

FuturICT will build on emerging research in areas such as Reality Mining and large-scale real-time analytics of social media to endow ICT with collective and selfawareness. A crucial element will be to combine research in ubiquitous sensing, machine learning and knowledge discovery with large-scale, complexity-science-based techno-socio-economic models.

Creating collective and self-awareness is also a key element of a beneficial coevolution of ICT and society. The aim is to enable the global ICT system to autonomously adapt to social needs, react to unforeseen events and generally have a desirable, stabilising effect on social processes and phenomena. The system will be able to reshuffle resources (information sources, bandwidth, distributed computing resources etc.) to enable better analysis and management of emerging crisis situations, to mediate interactions in and between communities, and to provide emergency "slow down and ask human" mechanisms, preventing the system from accelerating crises and systemic failures.

In summary, FuturICT will facilitate a paradigm shift from the focus on a single device reacting to its immediate environment to a systemic view on a global scale, in order to enable the understanding and proper response to complex techno-socioeconomic phenomena and collective behaviours emerging in our world. Such socially interactive systems will be controlled democratically by the people in a bottom-up way and implemented by building on favourable self-organisation processes, leveraging the systems' collective awareness and the ability to model complex social phenomena. 


\section{BOX 5: Research priorities}

FuturICT plans to build its ICT platform by integrating various Observatories to form interconnected Exploratories of Society, Economy, Technology, and Environment, which will eventually be fused to form a single, integrated infrastructure. The interconnected Observatories and Exploratories will feature real-time data-mining, theory-based computer simulations, systematic scenario analyses, and interactive environments allowing people to explore realistic virtual worlds.

Since the Observatories start off from already existing research cores, first practically relevant applications are expected after 2 years. Practical use cases of the FuturICT flagship include smart cities (as more than $50 \%$ of the world population is now living in cities), smart energy systems (as micro-generation of electrical power will increase the number of independent energy providers multiple times), smart health systems (to ensure a high quality of life in an ageing society at affordable costs), and better financial architectures (to reduce the related societal losses). New solutions will also be developed to reduce crime, corruption, and conflict. Eventually, FuturICT's Participatory Platform will inform decision-makers and involve citizens. A focus on Managing Complexity will develop integrative system designs and new decision-making and governance tools. The Innovation Accelerator will speed up research, development, and the creation of new business opportunities. Finally, a new wave of socially inspired ICT will be developed.

To gain a better understanding of social, economic and ICT systems, a number of over-arching research challenges must be addressed, such as:

1. the interrelationship between structure, dynamics, and function,

2. strongly coupled systems, interdependent networks,

3. contagion and cascading effects,

4. ecological and social systems approaches,

5. concepts to manage complexity,

6. suitable incentive schemes,

7. integrative systems design,

8. resilience,

9. systemic risks,

10. sustainability,

11. trust.

Over a ten years time period, FuturICT is expected to reduce current knowledge gaps by developing a global systems science, which will advance the following new research areas: the science of strongly coupled systems, a complexity science for real-world problems (including non-equilibrium economics), a new data science, and a systemic risk calculus. On the engineering side, the co-evolution of ICT with society and the development of socio-inspired technologies are challenging new areas. Presently, these areas are almost like new continents waiting to be discovered, and many new breakthroughs are expected.

\section{The urgent need of a federated big science approach}

The complexity in our ICT systems and the techno-socio-economic challenges of humanity in the $21^{\text {st }}$ century require our society to make a large-scale federated investment to counter-act disastrous cascading effects (see Appendix B), negative interferences, and tragedies of the commons, and fill the current knowledge gaps to enable this (see Box 5). The FET Flagship Call by the European Commission provides a unique and timely opportunity for this (see Appendix C). 


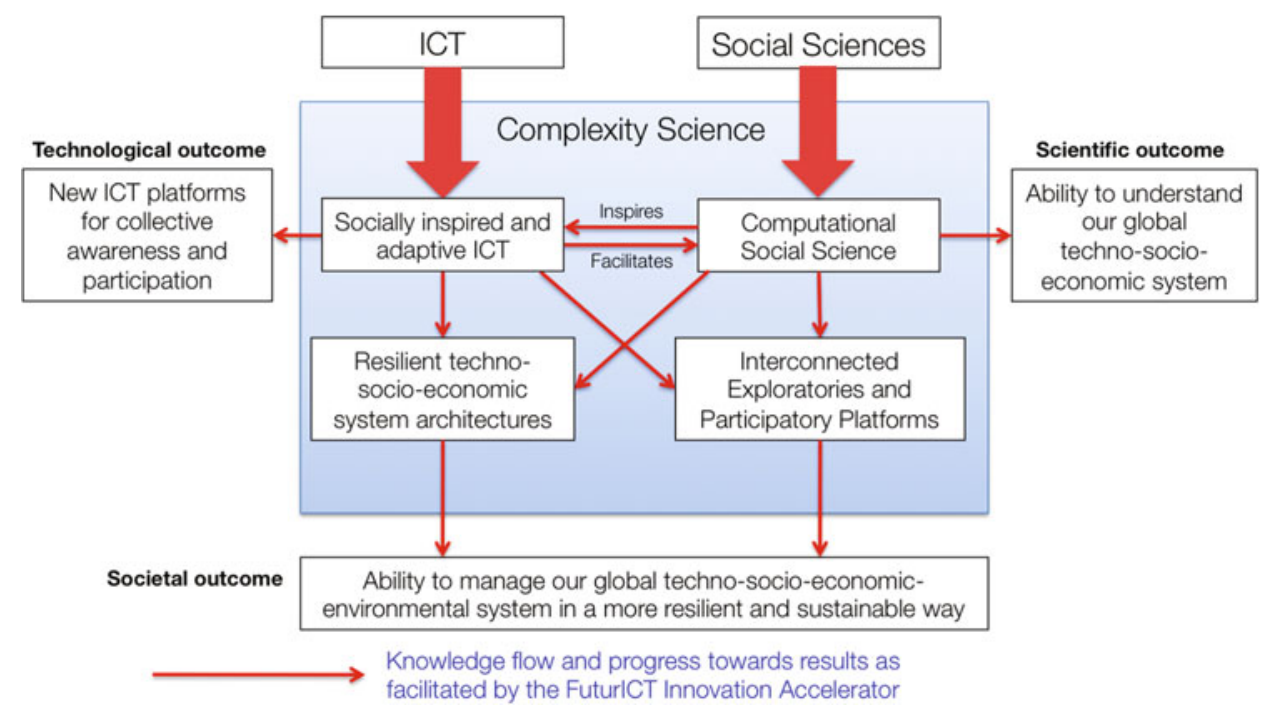

Fig. 1. The interdisciplinary concept of FuturICT foresees the integration of expertise in information and communication technology (ICT), complexity and social sciences, to create outcomes in science, technology, and society.

We urgently need to learn how to manage our future in a complex, strongly connected world. We, furthermore, need to develop realistic scientific models of our globalised techno-socio-economic world. A federated approach is ultimately required to extend the available capacities for systemic risk assessment and integrated risk management.

\section{Need for a new multi-level complex systems science}

The challenges of the $21^{\text {st }}$ century require the development of a new kind of complexity science: the science of multi-level complex systems focusing on realistic models rather than just metaphorical analyses. This new science will allow us to understand not only the impact of system components on others, but also the resulting links between micro-level interactions and macro-level behaviour (and vice versa).

We also need to understand the complex interdependencies between the different stakeholders, institutions, infrastructures and networks on which our techno-socioeconomic world is based. This requires social scientists in order to ask the right questions (including ethical ones), develop a characterisation of the system components (individuals, institutions, etc.), and provide a theoretical framework.

It requires complexity scientists to gain a systemic understanding by studying the interactions of the components and their emergent collective behaviour and organization. And it requires computer scientists as well as information and communication experts to develop new data acquisition and processing methods and platforms that will allow us to better understand and manage our world (see Fig. 1).

\section{Need for a new data science}

The FuturICT project also calls for a new Data Science (or a new "Social Information Theory"), which focuses not just on bits and bytes, but also on the meaning and impact of information (as we need to learn under what conditions new knowledge 


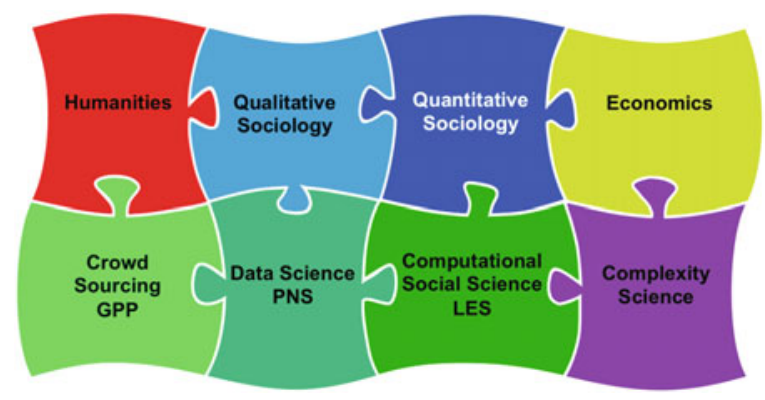

Fig. 2. The plan is to promote an innovation ecosystem integrating various social, natural, and engineering sciences, but also to advance emerging fields such as computational social science, a new data science, and a practically relevant complexity science. The close interaction of these fields will accelerate knowledge creation and facilitate the development of new platforms for the exploration of techno-socio-economic systems: the Global Participatory Platform (GPP), the Planetary Nervous System (PNS), and the Living Earth Simulator (LES).

is created from existing pieces of information). Moreover, it requires a considerable extension of complexity science to understand not only the stylised patterns and dynamics resulting from the non-linear interaction of simple elements, but also the result of interactions between individuals with cognitive complexity or system elements with a complex response to the surrounding world. Such systems with various levels of complexity are probably not analytically tractable anymore and, therefore, require the use of new modelling approaches and of future supercomputers.

Despite the urgent need for a global systems science and although many of its components have been developed in the past, a coordinated effort has not yet come about due to institutional obstacles and a lack of resources. The FuturICT project will, for the first time, integrate all necessary competencies by bringing together the best of all available knowledge from the engineering, natural and social sciences (see Figs. 2 and 3 ).

This new science will be boosted by the availability of vast amounts of data from a wide variety of techno-socio-economic systems. In fact, future sensor networks will produce more live stream data than can be stored or moved around. To make use of them, they must be aggregated "on the fly" - and in a privacy-respecting way (see Box 6). But these data could also be used to collectively form something like a "Planetary Nervous System", which would make it possible to create collective and self-awareness of the impact that our decisions and actions are likely to have on our techno-socio-economic-environmental system (see Box 7).

An ability to quantify the social impact of our actions will help us to avoid decisions that exploit or destroy the socio-economic fabric on which our society is built, for example, social capital such as solidarity and trust. It will eventually promote a more responsible behaviour, just as the measurement of the environmental footprint has done. Developing the ability to quantify the social footprint seems a particularly promising way to successfully establish sustainable systems.

\section{What FuturICT would like to do: A way forward, aided by information}

The complexity of modern technology lies far beyond the capacity of what the human brain can analyse and comprehend. Information technology can considerably expand 


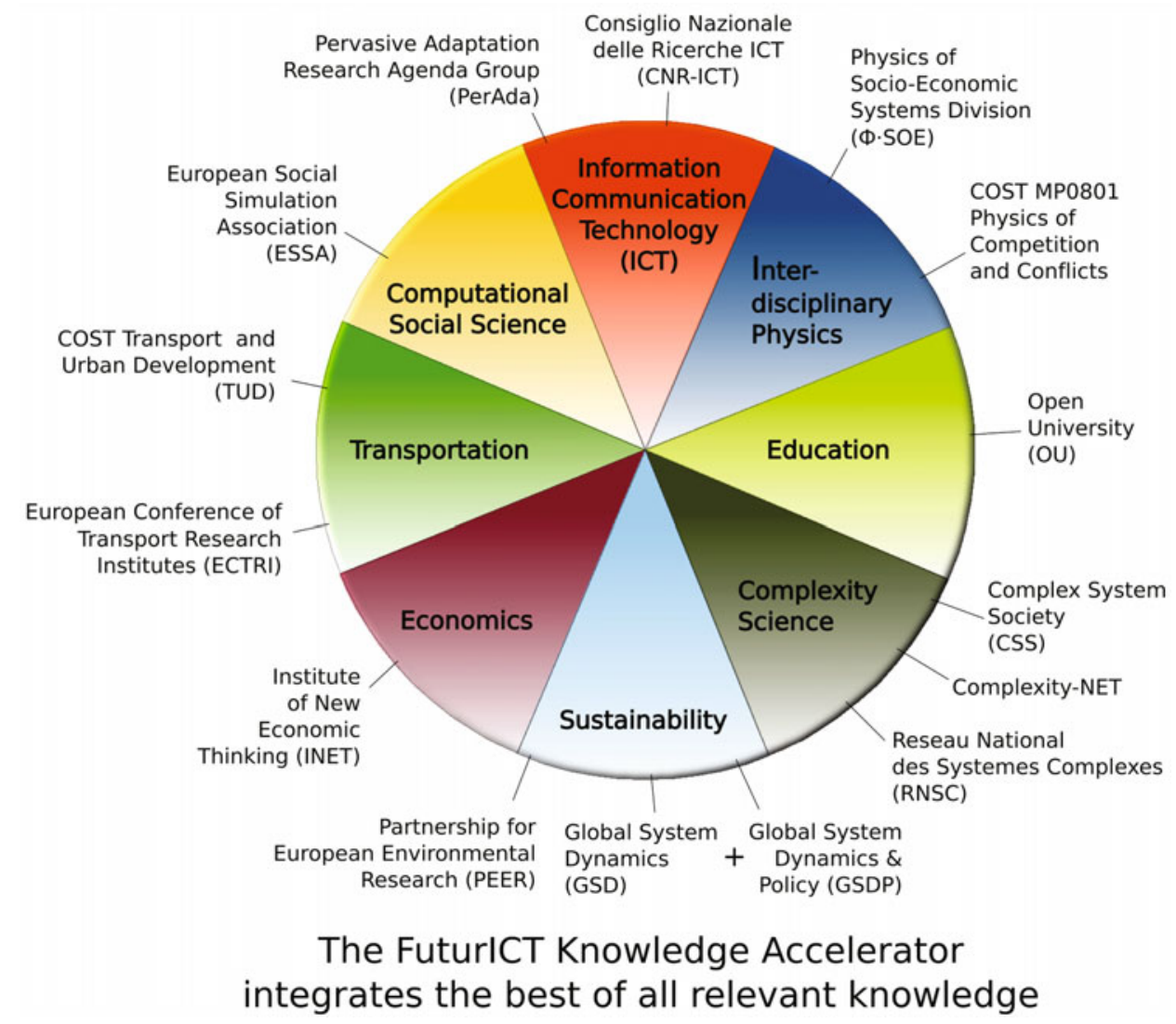

Fig. 3. FuturICT is a multi-disciplinary project integrating many different research areas. This is done with the support of various established science organisations, which stay in close contact with their respective research communities.

this capacity. For example, scientists and engineers rely on massive computer power and data processing to design and test everything from cars and electronic devices to medical drugs. We face an even greater complexity in our socio-economic systems, especially in the interaction with the rapidly expanding technological infrastructures such as the Internet and the Earth's vast, multi-component environment. Only recently, however, have we begun to exploit the power of information technology to gain a better understanding of the human-Earth system, and to improve our capacity to manage this system on the basis of well-founded knowledge.

The FuturICT project aims to develop new science and technology, capitalising on the current data revolution. The project will develop a visionary information platform, considering insights from the social sciences, complexity theory and ethics.

This system will be able to act as a "Policy Simulator" or "Policy Wind Tunnel", allowing people to test multiple options in a complex and uncertain world, and produce pluralistic perspectives of possible outcomes. The platform would analyse data on a massive scale and leverage them with scientific knowledge, thereby giving politicians and decision-makers a better understanding to base their decisions on.

Through the concept of a socially inclusive Participatory Platform, FuturICT will extend such capabilities to empower citizens, communities, small businesses, and nongovernment organizations, creating a whole ecosystem of new applications and forms of social and economic participation. In the long run, this would enable every one of 
us to explore the possible or likely consequences of even barely imaginable scenarios, effectively helping us to see just a little around the corner into possible futures (see Box 7). The potential benefits are huge: reducing the impact of major societal and economic problems by only 1 percent would save the European Union billions of Euros every year (see Box 8). Indeed, the social and complexity sciences can present a number of recent impressive success stories (see Box 2). Thus, similar to weather forecasts, it is expected that FuturICT can create value that is many times higher than the required investments.

\section{BOX 6: How to establish a planetary nervous system}

The goal of creating a planetary nervous system is to measure the state of the world and the interactions in it. For this, real-time data mining, so-called "reality mining", will be established, using data of the Internet and the semantic web. Additionally, data will be collected by linking sensors which aggregate information about the technological, social, or economic activities around them. Such a global sensor network can, for example, be established by connecting the sensors in today's smartphones (which comprise accelerometers, microphones, video functions, compasses, GPS, and more). Here, FuturICT will closely collaborate with Prof. Sandy Pentland's team at MIT's Media Lab [1]. In order to reach that users will contribute their own data voluntarily, a number of criteria must be fulfilled:

1. The system must provide incentives (such as sharing benefits and profits generated with the provided data).

2. A micropayment system is needed to establish a market for the reward points earned.

3. Users must be given control over their own data and what they are used for.

4. A privacy-respecting data mining approach must be developed.

5. Macroscopic measurement procedures must be invented to anonymise and aggregate sensitive data "on the fly".

Illustrative examples for smart-phone-based collective sensing applications are the open streetmap project [2], the decentralised earthquake sensing and warning system [3], and the reconstruction of our $3 d$ environment from publicly accessible photographs [4].

\section{How will it work?}

To achieve its goals, the FuturICT project will work out new conceptual, theoretical, methodological, and technological approaches. In particular, it will develop new information and communication technology (ICT) to collect massive data sets and mine them for meaningful and useful information. It will also have the capacity to selforganise and adapt to the collective needs of users. These ICT systems will be the basis of the FuturICT Platform (see Fig. 1). It will be built on three new inter-connected instruments to gain novel insights into our world: the "Living Earth Simulator", the "Planetary Nervous System" and the "Global Participatory Platform" (see Fig. 3).

The Living Earth Simulator will enable a model-based scenario exploration at different degrees of detail, integrating heterogeneous data and models and employing a variety of perspectives and methods (such as sophisticated agent-based simulations, multi-level models, and new empirical and experimental approaches). Exploration will be supported via a "World of Modelling" open software platform, comparable to an 
app-store, to which scientists and developers can upload theoretically informed and empirically validated modelling components that map parts of our real world.

\section{BOX 7: Possibilities and limits of prediction}

FuturICT is often confronted with questions regarding the predictability of its models [5-7]. Recent findings suggest that the dynamics in social systems depends at least on four different factors: the situational context, interaction effects, history, and random factors. Therefore, the measurement of the first three factors should, in principle, allow for probabilistic forecasts.

In fact, a systemic analysis, combined with a situational analysis, allowed one to predict developments such as the destabilisation of the financial system or the overall political impact of September 11 on various countries. Nevertheless, long-term forecasts are not a goal of FuturICT. We recognise that long-term forecasts are restricted to a few global trends (such as Moore's law or population growth and related resource issues), and that forecasting the exact timing is generally difficult in social systems. However, even when only probabilistic short-term anticipation is possible (as in the case of weather forecasts), this is often sufficient to achieve significant benefits by adaptive strategies (e.g. principles of self-organisation and self-control, see Box 4). In addition, it is often possible to predict likely courses of events, since cascading effects follow from causal relationships (see Fig. below). Note that an assessment of the robustness of a system does not require forecasting (i.e. when something will be happening), but only a predictive model (that says under what conditions something is likely to happen). Given suitable data, it increasingly becomes possible to determine how the state of a complex system depends on the properties of the system components, their interactions, the environment, institutional setting, and resources. Given the availability of situational and contextual data, it should also become possible to determine the impact of predictions on social systems, i.e. to identify when a self-defeating prophecy is expected to occur, or a self-fulfilling prophecy, or no significant effect at all. Finally, as has been experimentally demonstrated, it is possible to design recommender systems in such a way that their usefulness is not undermined by information feedbacks [8].

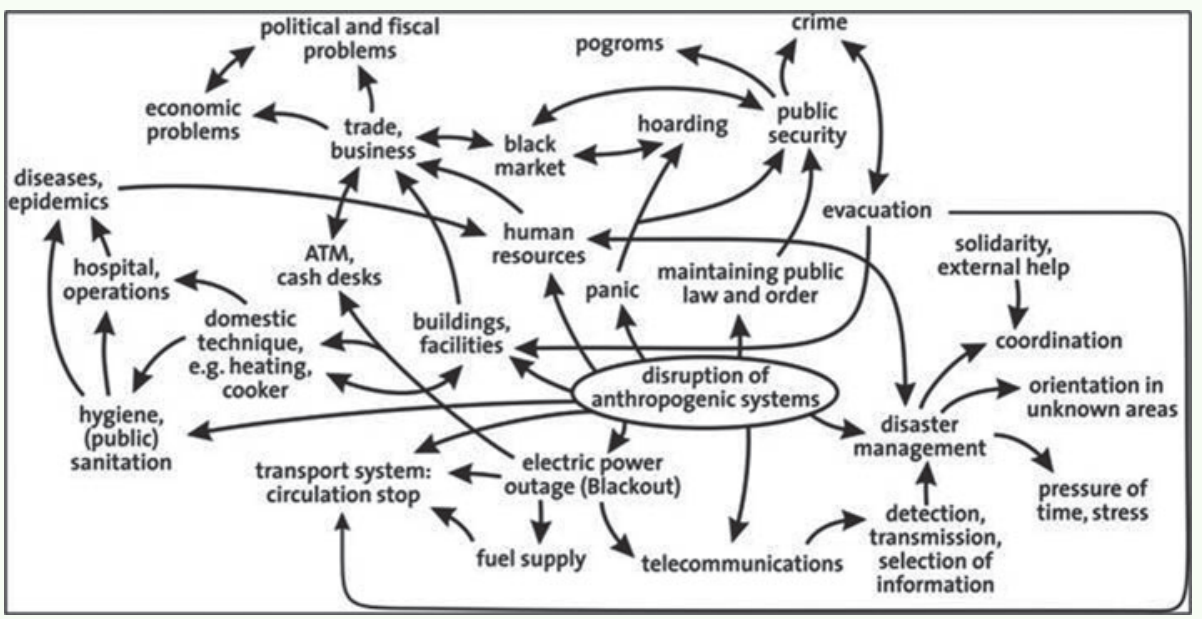

Illustration of cascading effects in techno-socio-economic-environmental systems, which may be triggered by the disruption (over-critical perturbation) of an anthropogenic system. A more detailed picture can be given for specific disasters. Note that the largest financial damage of most disasters is caused by such cascading effects. 


\section{BOX 8: Costs and benefits of the FuturICT project}

Among other challenges, FuturICT will develop new concepts to address the following problems, the societal costs of which are listed below:

1. Financial crisis: Losses between 2 and 20 trillion $\$$

2. Crime and corruption: $2-5 \%$ of GDP, about 2 trillion $\$$ annually.

3. Conflict: Global military expenditures of 1.5 trillion $\$$ annually.

4. Terrorism: 90 billion $\$$ lost output of the US economy as a result of $9 / 11$ attacks.

5. Flu: A true influenza pandemic infecting $1 \%$ of the world population would cause losses of 1-2 trillion $\$$ per annum.

6. Cyber-crime: 750 billion EUR damage each year in Europe.

7. Congestion: Impact of 7-8 billion in the UK alone.

If the impact of these societal problems would be reduced by $1 \%$ only, this would already create a benefit many times higher than the investments into the FuturICT Flagship project. Based on previous success stories regarding a better management of complex systems, we expect that an improved understanding of the fundamental underlying issues will facilitate improvements between $10 \%$ and $30 \%$, given the new insights are properly applied. For comparison: Swiss citizens pay 10 Swiss Francs per year for the public weather forecasting system, but the benefit is 5 times higher.

Significant benefits are also expected from ICT-related growth and productivity: ICT-producing industries contribute directly to productivity and growth through their own rapid technological progress. For example, a rough estimate indicates that in the United States, Internet intermediaries contributed at least $1.4 \%$ of GDP value added in 2008. This produces "spill-over effects" on the rest of the economy as ICT diffusion leads to innovation and efficiency gains in other sectors. A good example for the economic potential of socio-inspired ICT is the company Facebook, which is believed to have already a value of many billion US dollars. Further prospects and benefits of the FuturICT project are summarised in Box 13.

The Living Earth Simulator will require the development of interactive, decentralised, scalable computing infrastructures, coupled with an access to huge amounts of data, which will become available by integrating various data sources coming from online surveys, web and lab experiments, and from large-scale data mining (see Box 9 and Box 10).

This is where the Planetary Nervous System comes in. It can be imagined as a global sensor network, where "sensors" include anything (including the Internet) able to provide data in real-time about socio-economic, environmental or technological systems. Such an infrastructure will enable real-time data mining ("reality mining") and the calibration and validation of coupled models of socio-economic, technological and environmental systems with their complex interactions. It will even be possible to extract suitable models in a data-driven way, guided by theoretical knowledge.

The Global Participatory Platform will support communication, coordination, cooperation and social, economic and political participation of citizens beyond what is possible through the eGovernance platforms of today. In this way, FuturICT will create opportunities to reduce the gap between users and providers, customers and producers etc., facilitating a participation in industrial and social value generation. Building on the success principles of Wikipedia and the Web2.0, societies will be able to harness the knowledge and creativity of multiple minds much better than we can do today. The Global Participatory Platform will also support the creation of Interactive Virtual Worlds. Using techniques such as serious multi-player online games, 


\section{BOX 9: FuturICT's new approach to ICT systems and innovation}

Humans are a unique species, as their behaviour is largely driven by information. By the creation of Interactive Virtual Worlds and in many other ways, future ICT systems will partly overcome the limitations of our physical and biological world. In fact, they will create an almost unlimited number of new goods and services, and thereby many new economic opportunities, but also social and political ones.

Europe's vision of creating an Innovation Union is a logical response to these opportunities. With its Innovation Accelerator approach, FuturICT will contribute to filling this vision with life. Through new ICT technologies, Innovation Scouts and Knowledge Transfer Supply Chains, the distance between academic inventions and innovations in the technological, social and political realm will be significantly reduced. In this way, transforming new ideas into new products will be more efficient than today (currently this requires of the order of 30 years in many areas).

Fundamentally new ICT systems that are responsive, responsible, ethical and privacy-respecting by design, are key to exploring, understanding, and managing our future in a resilient and sustainable way. The FuturICT flagship will promote the required paradigm shifts:

- by integrating the best knowledge from the engineering, natural and social sciences, and bringing together Big Data, explanatory theories, massive computer simulations, and large-scale experiments in virtual and real-world settings, and

- by mutually empowering the social sciences and future ICT systems and employing a complex systems perspective to understand the interaction-based and institutional principles that make strongly connected, socially interactive systems work well.

This will trigger a new era of the social sciences and a wave of socio-inspired technologies (beyond social networking, the wisdom of crowds, and prediction markets). Future ICT systems will have the capability of social sensing, social thinking, social adaptiveness, social self-organisation, etc. They will feature ICT-based cultures, collective and self-awareness, reputation- and trust-based applications. And they will enable mixed realities, where it won't be possible to tell apart the virtual and real world. The goal will be a beneficial human-information symbiosis.

The creation of such an information ecosystem will require a new kind of complexity science, which is capable of realistically understanding multi-level complex techno-socioeconomic systems. This new complexity science will, in turn, facilitate the reduction of systemic risks by employing suitable decoupling strategies and the design of resilient and sustainable socio-economic and ICT systems. However, a well-functioning information ecology also needs to overcome deficits in transparency, accountability, quality standards and trustworthiness, which most free data services provided on the web currently have. At present, many companies collect huge data sets, but these are often fragmented and potentially sensitive. It is not clear to what extent they manipulate the users.

As the World Economic Forum points out [9], users should be given control over their personal data. Moreover, it is important to develop methods of privacy-respecting data mining, which can satisfy individual, commercial and public interests at the same time. For a proposal how to do this, see [10]). This will require a scalable bottomup approach, transparency, user control, encryption of sensitive data and digital rights management, plus a manipulation-proof reputation system that supports a healthy "immune response" to malicious data and activities. On this basis, it will be possible to develop design and operation principles of self-organizing, trustworthy, reliable and open ICT systems promoting responsible use. The creation of an integrated multi-disciplinary reputation-based science platform will be an important use case. 


\section{BOX 10: Big data: a new era of the social sciences is ahead}

In the past, getting data about social systems and social interactions was very time consuming and cumbersome. In the meantime, lab and web experiments and online surveys have simplified the collection of data, and the internet as well as other information and communication systems are collecting tons of data allowing one to study social activity patterns [1]. Recently, there are more bits of information than stars in the universe, and the volume of data grows by a factor of 10 every 5 years. This is opening up the door for a new era of the social sciences. In parallel, much progress has been made in modelling key elements of social systems. Now, there are models considering spatial and network interactions, heterogeneity, and randomness (which can change the systemic outcome dramatically). There are also models of emergence of cooperation under unexpected conditions (namely in social dilemma situations, which normally promote a "tragedy of the commons"), models for the formation of social norms (even when individuals have to make sacrifices for this), and models for the spreading of conflicts or violence, models of collective behaviour (such as opinion formation, crowd disasters, revolutions), as well as models taking into account communication and learning. Currently, scientists are working on models considering emotions, models explaining conditions for other-regarding behaviour, and models considering cognitive complexity. In perspective, these models will enable many beneficial applications for society, as Big Data become available to calibrate and validate them (see Box 2) [11].

we will be able to explore possible futures - not only for different designs of shopping malls, airports, or city centres, but also for different financial architectures or voting systems.

In addition to the interconnected systems forming the FuturICT Platform, the flagship project will also create an Innovation Accelerator that will identify innovations early on, distil valuable knowledge from a flood of information, find the best experts for projects, and fuel distributed knowledge generation through modern crowd sourcing approaches. In particular, the Innovation Accelerator will support communication and flexible coordination in large-scale projects, co-creation, and quality assessment. Hence, the Innovation Accelerator will also form the basis of the innovative management of the FuturICT flagship. Beyond this, it will fill the vision of Europe's Innovation Union with life by creating many new business opportunities based on socio-inspired and other new technologies (see Box 13).

It is time for Practical Steps to a Better Future To succeed with its ambitious endeavour, the FuturICT project team is building communities in most European countries and other continents, bridging between the so far separated areas of ICT, social and complexity sciences. It will build the FuturICT platform by integrating 4 interconnected Exploratories, which are ICT infrastructures to explore our global techno-socio-economic-environmental system through the combination of large-scale data mining, multi-level modelling, supercomputing and participatory approaches.

The Exploratories of Society, Economy, Technology, and Environment are composed of various interactive Observatories, for example, Observatories of Financial and Economic Systems, of Conflicts and Wars, of Social Well-Being, of Health Risks, of Transportation and Logistics (see Fig. 4). Integrating the Observatories and Exploratories over time will overcome disciplinary silo thinking and eventually facilitate a systemic picture of risks and opportunities as well as integrated risk management. Note that FuturICT will pursue a pluralistic approach, allowing people to 


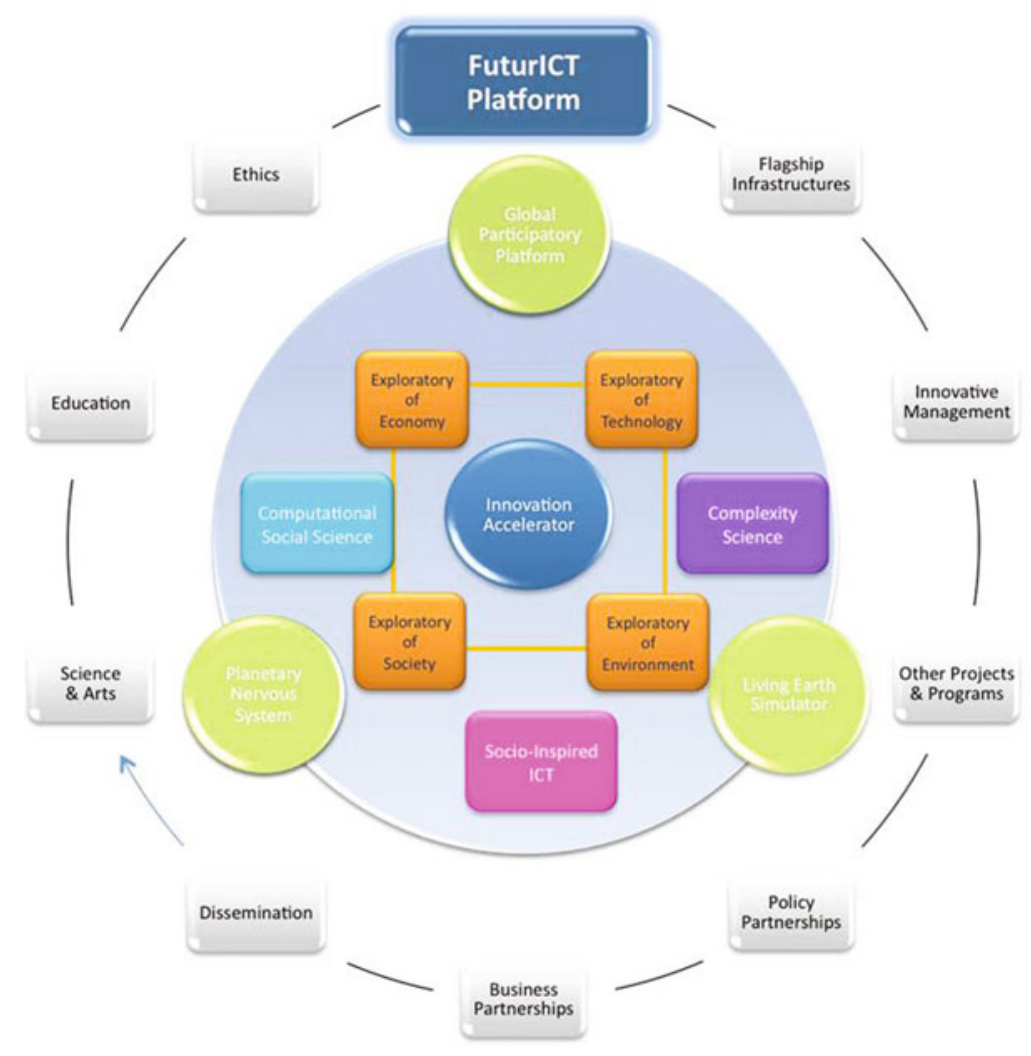

Fig. 4. Main components and activity areas of the FuturICT flagship project.

study many different perspectives in parallel. This will provide a more differentiated picture of the interactions on our planet and allow us to better orient ourselves and manage our way forward in a rapidly changing world.

\section{Potential and need of socio-inspired technologies}

FuturICT's research program will also be crucial for the effective design of future ICT systems, since these are indeed becoming socially interactive systems (see the Section on Artificial Social Systems above and Fig. 5). As our society is now largely dependent on information and communication technologies, their stability and reliability has become absolutely crucial but at least for current designs, this stability is not guaranteed. Systemic breakdowns, cyber-crime and cyber-war are problems that have recently become virulent and show the vulnerability of the systems to cascading failures and other problems (see Appendix B). At the same time, several social features such as self-organisation, adaptiveness, emergent cooperation, social norms, cultures and community formation are new attractive features of future ICT systems. Trust is just one example of a hardly understood, but crucial property of social systems. The creation of principles for trustworthy ICT systems, based on principles of social, reputation-based self-control to keep cyber-crime down, is an important example of future socio-inspired technologies. 


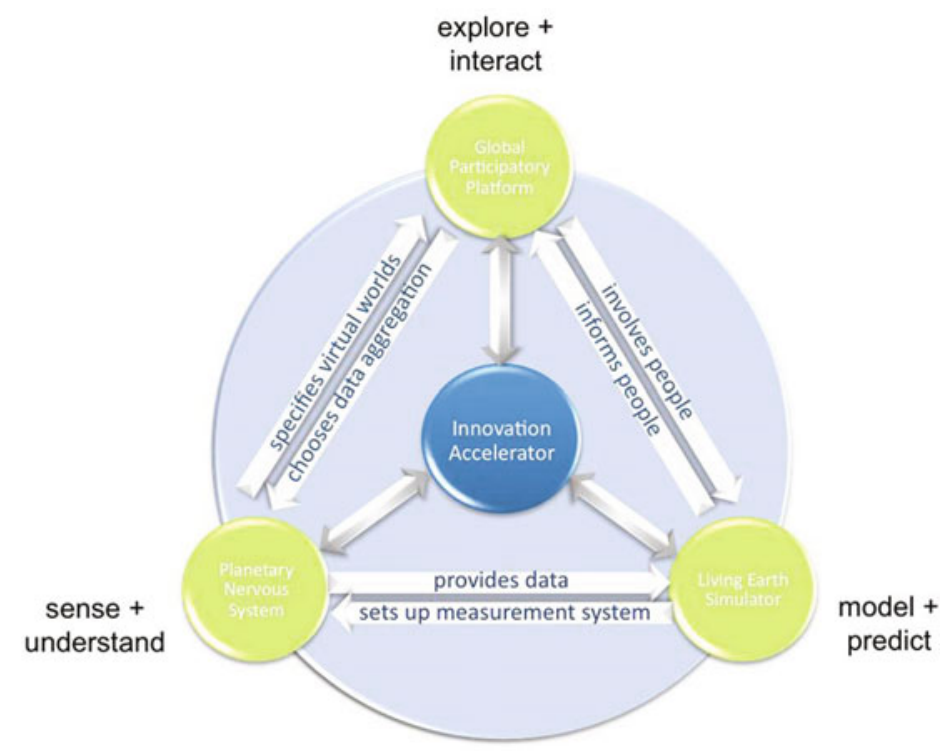

Fig. 5. Interdependencies between FuturICT's main ICT components. The project will develop 3 key instruments to study our techno-socio-economic-environmental world: The Living Earth Simulator, the Planetary Nervous System, and the Global Participatory Platform. Analogously to microscopes and telescopes, they may be considered to establish the 'socioscopes' and socio-economic-political compasses of the future.

\section{What FuturICT does NOT plan to do}

Most importantly, the FuturICT project will NOT attempt to collect "all the data in the world", or to represent each individual on the globe by an identical copy in some giant multi-agent simulation, considering private features and preferences of all individuals. Science is the art of abstraction and approximation. Just as maps do not show all the features of our environment, a scientific model is specified in such a way that a particular question can be addressed in the simplest possible manner.

That is, models are to be problem-specific, and parameters and variables not expected to be relevant for the answer should be neglected. In many cases, one is interested in global interdependencies on the aggregate level. Then, a macroscopic description is sufficient. Most computer simulations are based on a multi-level approach and not on the micro-simulation of the individual system elements.

As a matter of principle, it would obviously not be possible or desirable to simulate each individual in detail, considering also the complexity of its cognitive dynamics. However, the interaction of many system elements often reduces the dimensionality of the relevant system dynamics (i.e. there is a small fraction of variables that matter, while many variables do not change over the relevant time scale and others change so quickly that they may be treated as random variables).

In other words, the largest amount of complexity seems to occur on the "microlevel" of the individual, while collective behaviour tends to be simpler due to many factors such as herding effects, social conventions, norms, contracts, and laws (otherwise we would not have cultural trends, fashions etc.). This implies the possibility of probabilistic short-term forecasts similar to weather forecasts (see Box 7). These are sufficient for adaptive management approaches, which can reach considerable improvements. 


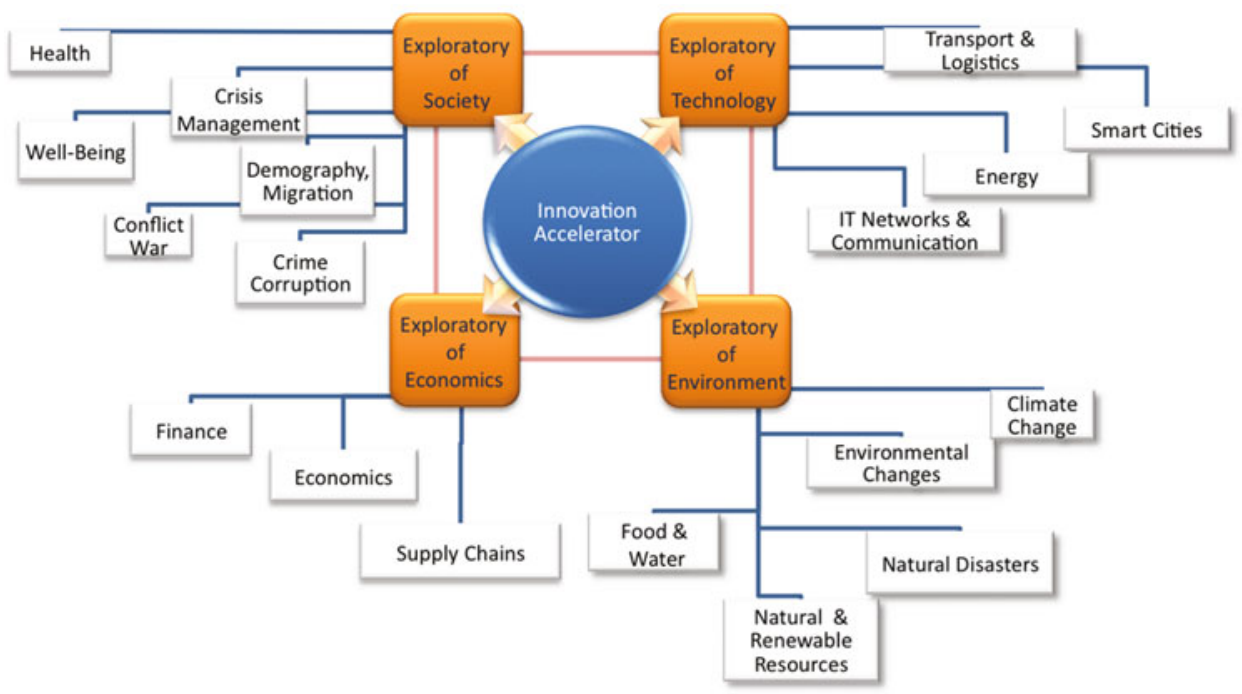

Fig. 6. The Exploratories of Society, Economy, Technology, and Environment are established by integration of various Observatories. The Exploratory of Society, for example, could be composed of Observatories for Social Well-Being, Health, Crime and Corruption, Conflict and War, Demography and Migration, and Resilience.

\section{Organisational principles}

Setting funding issues aside, the strategy of the Partners of the currently running FuturICT Pilot project is to formulate a visionary goal, to elaborate an ambitious project (as expected by the FET flagship program), to identify the related grand scientific challenges, to develop a research strategy and roadmap, to form an integrated multi-disciplinary community, and to develop a platform for global collaboration and exchange (see Figs. 6 and 7).

The FuturICT project is now supported by a large, and quickly growing multidisciplinary community (see Box 11) so that it is already aligning the research agendas of hundreds of scientists. FuturICT enjoys support in many European countries as well as in USA, Asia, and Australia. Step by step, the project will also create links to South-American and African countries.

In several non-European countries, there is a strong desire to participate in addressing the global challenges FuturICT will tackle, based on complementary national budgets. Therefore, FuturICT is trying to create an open platform with interfaces that would allow other countries and projects to team up. One may imagine this similar to the organisational concept of the International Space Station. FuturICT's prepatory activities have already changed and will continue to change the research landscape, and first spin-off companies have been established. Openness is an important organisational principle of FuturICT. We envisage that the composition of the consortium of experts will continuously change over time to take new rising stars of science on board. Through open calls, FuturICT plans to allocate substantial research budgets to innovative research in order to reward excellent findings and support future research activities.

Openness will also be achieved by creating interfaces with business partners and policy-makers. In particular, the research infrastructures created by FuturICT (such as the Exploratories of Society, Economics, Technology, and Environment) will be open to researchers from outside the FuturICT consortium. 


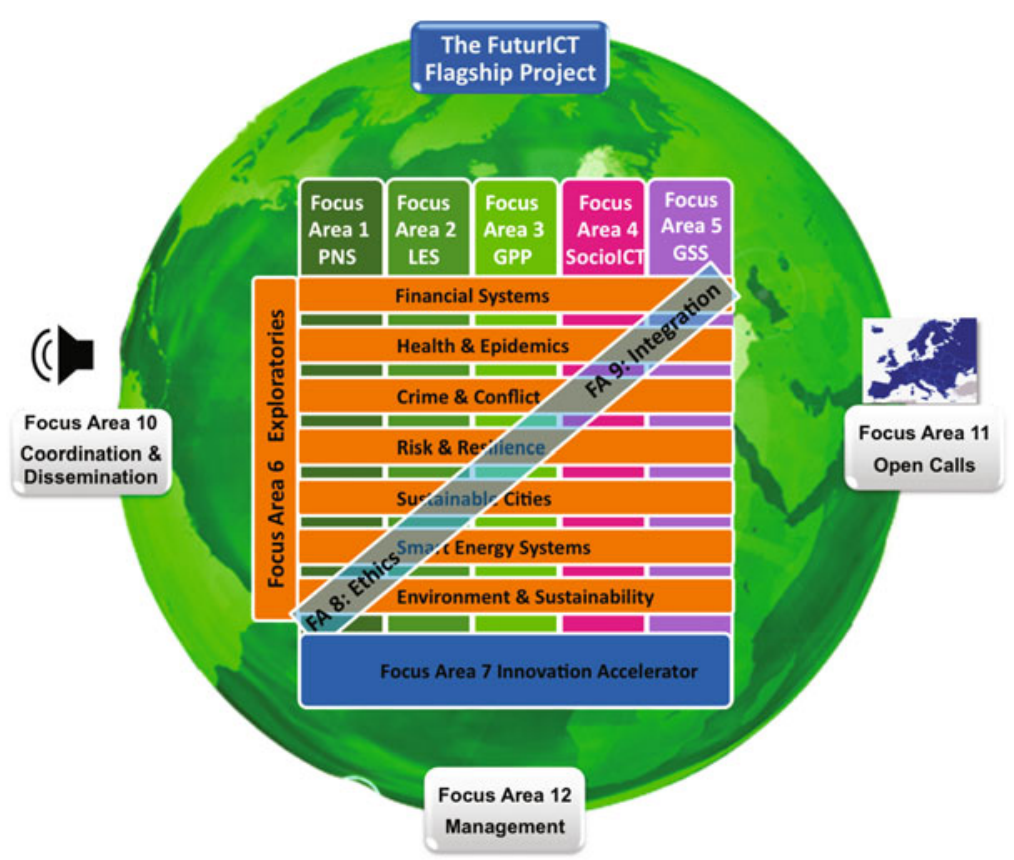

Fig. 7. Illustration of the tentative structure of the FuturICT Flagship activities. The project is organized in 12 closely interacting Focus Areas (FA). Vertical Focus Areas have a strong focus on fundamental aspects of research and ICT development. They include the development of the 3 main ICT components of the FuturICT Platform, namely the Planetary Nervous System (PNS), the Living Earth Simulator (LES), and the Global Participatory Platform (GPP), furthermore, the development of Socio-Inspired ICT (SocioICT), of a Global Systems Science (GSS), and an Innovation Accelerator (IA). The Exploratories (Focus Area 7) will be the outcomes of horizontal and more application-oriented activities. They integrate the elements of the vertical activities, in particular data mining, scenario simulation, and interactivity. Ethical research activities (Focus Areas 8) take place across all Focus Areas. The same applies to Integration activities (Focus Area 9). The Flagship project is supported by Coordination and Dissemination activities (Focus Area 10), a Staircase to Excellence (Focus Area 11), which is linked to FuturICT's Open Calls, and finally the Management of the FuturICT Flagship (Focus Area 12).

This concept and the multi-level structure of FuturICT are also designed for a steady expansion. In fact, scalability is an important organisational principle of the project, as it is anticipated that there will be an increasing demand for research in the area represented by FuturICT, i.e. the research platform must be suited to support continuous growth and participation.

FuturICT will have a flexible and modern organisation, based on a multi-level hub-and-spoke network, integrating bottom-up elements. Besides the subject-level organisation of FuturICT's research activities, FuturICT will build on strong national and regional FuturICT communities, often integrating dozens of teams from the areas of ICT, complexity science, and the social sciences.

These communities will help to stimulate and coordinate research activities beyond what the FuturICT project itself can fund, i.e. they will form a Network of Excellence and coordinate various activities that do not belong to the project directly, but relate 


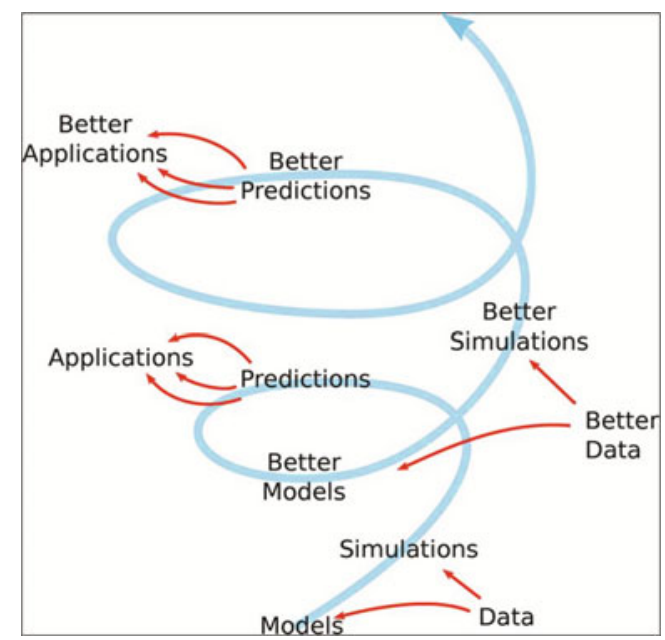

Fig. 8. Illustration of how Big Data of techno-socio-economic-environmental systems will boost better and better models, simulations, predictions, and applications. These will trigger off new business opportunities and spin-offs.

to it. The national scientific communities will be involved:

1. through annual national and international workshops (part of which will have a "Hilbert format", i.e. identify open problems and possible solution approaches rather than just presenting progress reports).

2. through awarding prizes to researchers for the best and most relevant results (providing money for follow-up research through the instrument of Open Calls).

\section{BOX 11: FuturICT's partners and supporters}

By now, FuturICT is supported by more than 1000 scientists worldwide. The project involves Europe's main academic powerhouses, such as ETH Zurich, University College London (UCL), Oxford University, the Fraunhofer Society, the Consiglio Nazionale delle Ricerche (CNR), the Centre National de la Recherch Scientifique (CNRS), Imperial College, etc. Several supercomputing centres support FuturICT. There are also letters of support by more than 100 academic institutions, organizations, and companies [12].

The list includes the OECD, the Joint Research Centre (JRC), regulatory authorities, international companies, and notable individuals such as George Soros. Furthermore, FuturICT has managed to integrate many different research communities, as the pie chart of Fig. 3 illustrates. In fact, the leaders of FuturICT have a long track record of successfully integrating scientists across disciplinary boundaries, as is reflected by dozens of multi-disciplinary workshops in the past years. FuturICT's supporters have also been involved in hundreds of successful projects with business partners.

The various academic institutions involved in FuturICT will typically receive their financial support through a few national institution(s). Matching funds from European countries and academic institutions will complement European funds and vice versa. 


\section{Summary and conclusions}

FuturICT is not starting from scratch. It can stand on the shoulders of giants and has learned from the experience of previous approaches (see Appendix D). Building on several hundred teams of scientists in Europe and all over the world (see Box 11), FuturICT has a strong potential to promote a beneficial co-evolution of ICT and society, and also to encourage a new synthesis in and with the social sciences supported by a plethora of computational methods for modelling, theory building, and real-time analytics.

Among other recent developments, the availability of Big Data will boost phenomenal progress in the social sciences (see Box 10), and will promote a new data science. Furthermore, creating an open platform, a "data commons" (see Box 12) is expected to trigger off a new era of information and communication technologies, services, and products (see Fig. 8 and Box 9).

\section{BOX 12: Creating an open, transparent platform for everyone}

FuturICT wants to overcome the current data fragmentation and "black holes" for data. It is trying to create an open platform for everyone. This includes establishing transparency regarding the data sources and their quality, the exact algorithms used, the statistical assessment of the results. Furthermore, it will be important to establish transparent, responsible use - a subject worked on by FuturICTs ethics committee. The result will be a new public good, like our environment, air, languages, and the Internet. This will enable an ecosystem of new services and jobs, and an age of creativity. The goal is to remove barriers for social, economic, and political participation. However, a public good requires measures to prevent a "tragedy of the commons", such as data pollution, manipulation, misuse, and cyber-crime.

In order to build trustworthy ICT systems, one must ensure in particular the control of users over their own data and the way they are used (see Box 13). One needs to create privacy-respecting information systems (and, hence, the use of pseudonyms). To promote responsible behaviour, a decentralised, transparent, and manipulation-resistant reputation system for information providers and contents is required, together with a proper incentive system. This will establish a self-organising and self-regulating system, something like a socially adaptive and mutually beneficial information ecosystem. To design the system properly, we need to understand socially interactive systems, otherwise one will end up with the same problems as in our society, i.e. instabilities, coordination failures, poor system performance and tragedies of the commons, conflicts, (cyber-)crime, and (cyber-)wars.

The main innovations of FuturICT may be summarized as follows:

- Integration of best knowledge from the natural, engineering, and social sciences, particularly ICT, social and complexity science to understand socially interactive systems.

- Building a global systems science, a systemic risk calculus.

- Enabling global, interactive reality mining from heterogeneous data across different temporal and spatial scales.

- Integrating heterogeneous data, models, algorithms, and services (foundational FuturICT resources).

- Developing a new data science: Bringing data and models together. 
- Creating a data and model commons.

- Empowering non-digitally-native users to engage, use, and contribute to the global body of knowledge.

- Learning how to manage complexity and how to do integrated systems design in a sustainability- and resilience-promoting way.

- New platforms and methods for Big Data business (European leadership)

- Developing a new (European) ICT paradigm:

- Socially interactive ICT

- Platforms for collective awareness

- Socio-inspired ICT: new paradigms for self-organization, adaptation and trustworthiness

- Ethical, value-sensitive, culturally fitting ICT (responsive and responsible)

- Participatory platforms allowing new forms of information sharing, collaboration, and political participation

- Co-evolution of ICT with society

- Instruments for knowledge creation

- Experimental and simulation platforms

- Privacy-respecting mining of Big Data

- A new information ecosystem

- Democratic control

- Mechanisms for user-centric co-creation of value.

\section{BOX 13: Some reasons to publicly invest into the FuturICT flagship project}

1. Considering what is at stake (see Boxes 3 and 12), we have a moral responsibility to undertake any effort needed to address the $21^{\text {st }}$ century challenges of humanity.

2. A federated Big Science approach is needed to catch up with the pace at which the world is changing and with the new political, social, economic and technological problems that are emerging.

3. FuturICT will provide policy- and decision-makers with innovative methods and instruments to improve the societal, economic, and political situation, while commercial companies do not have this capacity at present.

4. FuturICT will create new disaster reduction and recovery concepts for social, economic, technological and natural catastrophes.

5. FuturICT is an ethically oriented project and builds bridges between many scientific communities, which have previously worked in separation.

6. FuturICT can serve as a major driving force for all scientific research in the areas of ICT, social sciences and complexity science. It is triggering off entirely new trends in research and development, even beyond the research activities funded by the project. FuturICT will publish its results to benefit everyone, while private companies tend to keep their data, methods, and results for themselves.

7. FuturICT pursues an open platform approach under European leadership, which will allow other countries to participate (e.g. Japan, China, Singapore, Australia, South America, Africa).

8. FuturICT supports cooperative behavior on a global scale, while companies mostly tend to engage in competition.

9. FuturICT creates outcomes that profit-driven companies are unlikely to produce (such as privacy-respecting data mining technologies or an open data platform providing a high-quality public good).

10. It must be avoided that powerful tools and social innovations end up in the hands of a few stakeholders rather than benefiting humanity. 
Possible Success Criteria of the FuturICT project would be:

- PNS: Ability to interactively answer queries about our complex techno-socioeconomic system on a global scale, e.g. about factors relevant for social well-being.

- LES: Establishment of an Open World of Modelling Platform; financial market simulation, more resilient financial architecture.

- GPP: Mechanisms making PNS and LES features accessible to non-experts; an ecosystem of novel applications; an open education platform.

- Socio-inspired ICT: Novel design and operation principles for self-organizing, trustworthy, reliable and open global ICT systems promoting responsible use.

- Innovation Accelerator: Better indices to measure scientific value generation, number of spin-offs.

- Global Systems Science: Better understanding of systemic risks, models to describe how the interaction of information sparks off new trends, efficient methods to model and simulate real-world complex systems.

- Exploratories: Integration of data, models, and interactivity, e.g. new platform for behavioral experiments and simulation.

- Ethics: Development of principles of privacy-respecting data mining and of valuesensitive ICT that promotes responsible use; public ethical debate about challenges of Big Data and the Information Age.

The FuturICT Knowledge Accelerator will bring about a quantum leap in our capacity to more effectively cope with the speed at which our world is changing, and make a vital contribution to societal resilience and a sustainable future. It will do so by combining the best established scientific methods with multi-scale computer modelling, social supercomputing, large-scale data mining and participatory platforms (including web experiments and Interactive Virtual Worlds). Innovations needed to drive FuturICT forward to reach these ambitious goals will be promoted through a series of "Hilbert Workshops", i.e. think tanks to identify the fundamental problems and how they can be solved. As a result, we expect to see an era of social and socioinspired innovations, opening up new social, economic, and political opportunities. Indeed, there are many more reasons to make public investments into the FuturICT project (see Box 13).

\section{Further information}

Webpage (including publications, media response, events etc.): www.futurict.eu

Facebook (including videos): http://www.facebook. com/FuturICT

Twitter (including interesting quotes): https://twitter.com/FuturICT

Vimeo Video Channel: http://vimeo.com/futurict/videos

Visioneer White Papers: http://springerlink.metapress.com/content/1951$6355 / 195 / 1 /$

The authors are extremely grateful to the FuturICT community for interesting discussions, the Advisory Board for useful feedback, and the following people for valuable inputs: Eshel Ben-Jacob (particularly Box 13), Giulia Bonelli, Mark Buchanan, George Kampis, Kai Kunze (particularly Appendix B), Suzy Moat, Andrzej Nowak, Tobias Preis, Rob Procter, Janet Smart, Hilary Woodard (particularly Box 8), and others. The illustrations were produced by Stefano Balietti, Thomas Michael Bohnert, Peter Felten, George Kampis, Michael Mäs, Pratik Mukerji, and Stefan Lämmer.

The publication of this work was partially supported by the European Union's Seventh Framework Programme (FP7/2007-2013) under grant agreement no. 284709, a Coordination 
and Support Action in the Information and Communication Technologies activity area ("FuturICT" FET Flagship Pilot Project).

\section{Appendix A. FuturlCT's values}

FuturICT wants to promote human well-being, increase collective and self-awareness, reduce vulnerability and risk, increase resilience (the ability to absorb societal, economic, or environmental shocks), reduce damages due to large-scale loss of control related to unexpected cascading effects and systemic shifts, develop contingency plans, explore options for future opportunities and challenges, increase sustainability, facilitate flexible adaptation, promote fairness and happiness, protect and increase social capital, support economic, political, and social participation, find a good balance between central and decentralised (global and local) control, protect privacy and other human rights, pluralism and socio-bio-diversity, and support collaborative forms of competition ("co-opetition").

\section{Appendix B. Examples of cascading effects}

In 2011 alone, three major cascading effects have occurred, which may change the face of the world and the global balance of power: The financial crisis, the Arab spring and the earthquake, tsunami and nuclear disaster in Japan. In the following, we discuss some examples of cascading effects in more detail.

On November 4, 2006, an electricity line was temporarily turned off in Ems in Germany to facilitate the transfer of a Norwegian ship. This caused a chain-reaction leaving major parts of Europe without electricity. The previous scenario analysis had not checked for the coincidence with a possible spontaneous failure of another power line.

On December 22, 2010, Skype disseminated a faulty auto-update of its Internet telephony software. This led to a crash and reboot of most Skype super-nodes, a crucial part of their distributed system. To make things worse, the reboot of the super-nodes launched a distributed denial of service attack on the central Skype servers, thereby affecting worldwide data traffic.

Heavy solar storms, as expected for the near future, could simultaneously bring down major parts of the worldwide ICT system, since most ICT systems are not sufficiently shielded against the related atmospheric currents of electrically charged particles. As a consequence, cash machines, sales and customer supplies, computer and communication systems could fail critically at the same time in large areas.

The current economic crises started locally, due to a bursting real estate bubble in the US. The mortgage crises eventually hit building companies and caused the bankruptcy of more than 400 US banks. In the meantime, the financial, economic and spending crisis endangers the stability of the European currency and even of the European Union. Several countries (including Greece, Ireland, Portugal, Spain, Italy and the US) are at the verge of bankruptcy. If the crises cannot be stopped, this will probably cause social unrest, political extremism and increasing crime and violence, which even has the potential to endanger peace and the cultural foundations of our society.

The 2011 Tohoku earthquake in Japan caused a tsunami that triggered chain reactions and nuclear disasters in several reactors at Fukushima. Soon after, several countries, including Germany and Switzerland, decided to exit nuclear energy generation over the next decade $(\mathrm{s})$. However, alternative energy scenarios turn out to be politically vulnerable. Two of three major regions currently providing Europe with 
gas may not be entirely reliable. Moreover, Europe's DESERTEC project, a planned 1000 billion EUR investment into infrastructure to supply solar energy for Europe - has now an uncertain future due to another political unexpected event, the Arab Spring. This was triggered in particular by high food prices, which were no longer affordable to many people. These high food prices resulted partly from biofuel production, which intended to improve the global $\mathrm{CO}_{2}$ balance, but competed with food production. The increasing food prices were further amplified by financial speculation.

The spreading of flu outbreaks is very much promoted by worldwide travel and sometimes food supply chains as well. In the case of the resulting pandemics, economic and social life can be enormously affected (see Box 8).

\section{Appendix C. The European flagship program and its call for big science}

The FuturICT project is the response to a call from the Future and Emerging Technology (FET) section of the European Commission, therefore the name FET Flagship. The objective is to support Big Science in Europe with a "Man on the Moon" type of vision. In the first round, 21 flagship candidates were narrowed down to six Flagship Pilots.

FuturICT was determined as leading Flagship Pilot proposal addressing technosocio-economic and environmental challenges of the future.

Each pilot will submit detailed proposals in October 2012. At least two flagship projects will be supported by an amount of up to 1 billion EUR each over a time period of 10 years. This is about a tenth (or less) of what is invested into other Big Science projects: the CERN elementary particle accelerator, the ITER fusion reactor, or the Galileo satellite program, the Human Genome project, nanotechnology, etc. Approximately half of the money, i.e. 50 million EUR per year, must be mobilised by the project partners from national budgets and funding agencies, from business and industry, or from donations. It is planned to distribute a considerable fraction of the flagship budget through Open Calls. This will allow a wide scientific community to contribute to the common goals of the project.

What Distinguishes FuturICT from Other Candidate Flagships?

- FuturICT will develop a new science of Big Data, allowing one to understand how information is turned into useful knowledge, and under what conditions it impacts our real world (e.g. through game-changing innovations and new business opportunities).

- FuturICT will build a Planetary Nervous System by harvesting the data streams from smart sensors that are now becoming commercially available and spreading all over the world.

- FuturICT aims at building up non-embodied artificial intelligence, supporting collective (self-) awareness, and connecting the brains of millions or even billions of people to promote creativity and collective intelligence.

- FuturICT will promote social well-being. As humans prefer to be helped by others, the project also seeks ways to promote the mutual understanding and solidarity in increasingly multi-cultural and fragmented societies.

The particular strengths of the FuturICT project are its great societal relevance, the immediate importance of its results to master our everyday life in the future, its large and quickly growing community integrating multiple disciplinary backgrounds, the participation of many European countries, the significant support of scientific communities in other continents, the strong focus on ethical issues, the open project architecture, the innovative organisational concept with its bottom-up elements, and 
the remarkable activities in the area of education. Further benefits of FuturICT are discussed in Box 13.

\section{Appendix D. History and reference cases}

A strong historical backdrop is provided by the Digital Earth project, see http://www.digitalearth-isde.org/ and http://en.wikipedia.org/wiki/ Digital_Earth. 2011

The following quotes are from the above Wikipedia page, accessed on July 24,

"In a speech prepared for the California Science Center in Los Angeles on January 31, 1998, the former US vice president Al Gore described a digital future where schoolchildren - indeed all the world's citizens - could interact with a computer-generated three-dimensional spinning virtual globe and access vast amounts of scientific and cultural information to help them understand the Earth and its human activities..."

Digital Earth has come to stand for the large and growing set of web-based geographic computing systems worldwide. These are both useful and promising, but do not yet constitute the envisioned "global commons".

Below follow two excerpts from the Beijing Declaration on Digital Earth http://159.226.224.4/isde6en/hykx11.html:

"Digital Earth is an integral part of other advanced technologies including: earth observation, geo-information systems, global positioning systems, communication networks, sensor webs, electromagnetic identifiers, virtual reality, grid computation, etc. It is seen as a global strategic contributor to scientific and technological developments, and will be a catalyst in finding solutions to international scientific and societal issues."

"Digital Earth should play a strategic and sustainable role in addressing such challenges to human society as natural resource depletion, food and water insecurity, energy shortages, environmental degradation, natural disasters response, population explosion, and, in particular, global climate change."

Considering this, FuturICT may be seen as a logical continuation of the Digital Earth Agenda with a focus on:

1. exploring and managing techno-socio-economic systems

2. real-time mining and modelling of techno-socio-economic data to promote collective (self-) awareness, and

3. creating participatory platforms including Interactive Virtual Worlds.

Selected reference cases further illustrating FuturICT's feasibility include:

- IBM SmarterPlanet: http://www.ibm.com/smarterplanet

- Earth Simulator: http://www.jamstec.go.jp/esc/index.en.html

- Microsoft Modeling the World: http://www.modelingtheworld.com/

- PAX Early Warning of Conflict: http://www.pax2011.org/index.php

- Sentient World: http://www.scribd.com/doc/25656152/Sentient-WorldSimulation

- Planetary Skin: http://www.planetaryskin.org/

- Second Life: http://secondlife.com/

- Google.org: http://www.google.org/

- Gapminder: http://www.gapminder.org/

- Observatorium: http://www.observatorium.eu 


\section{Appendix E. How FuturICT differs from previous approaches?}

There have obviously been previous attempts to model the dynamics of the world. FuturICT has learned from them. It has a more sophisticated and more differentiated approach and is supported by hundreds of scientists worldwide.

FuturICT is not aiming at long-term forecasts. It addresses current and generic problems. It aims at improving the system performance and the ability to absorb shocks (see Box 5 for its other goals).

FuturICT's models will consider spatial and network effects, heterogeneity and randomness. They will build on the availability of Big Data and the possibility of real-time data mining as well as the progress in network theory, complex systems theory, multi-agent simulation, multi-level modelling, computational social science, experimental approaches and interactive platforms. Furthermore, FuturICT will develop new methods of investigation such as interconnected Exploratories, a Living Earth Simulator, a Planetary Nervous System, and a Global Participatory Platform.

\section{References}

1. Sandy Pentland, Society's Nervous System: Building Effective Government, Energy, and Public Health Systems (2012) http://hd.media.mit.edu/tech-reports/TR-664.pdf

2. M.F. Goodchild, GeoJournal 69, 211 (2007)

3. M. Faulkner, et al., The next big one: Detecting earthquakes and other rare events from community-based sensors (2011) http://las.ethz.ch/files/faulkner11next.pdf

4. J.-M. Frahm, P. Fite-Georgel, D. Gallup, T. Johnson, R. Raguram, C. Wu, Y.-H. Jen, E. Dunn, B. Clipp, S. Lazebnik, M. Pollefeys, Proceedings of the $11^{\text {th }}$ European Conference on Computer Vision, Part IV (ECCV'10), edited by K. Daniilidis, P. Maragos, N. Paragios (Springer, Berlin, Heidelberg, 2010), p. 368

5. D. Helbing, S. Balietti, EPJ Special Topics 195, 3 (2011)

6. D. Helbing, S. Balietti, EPJ Special Topics 195, 69 (2011)

7. How to do agent-based simulations in the future: From modelling social mechanisms to emergent phenomena and interactive systems design Santa Fe Working Paper 11-06024 (2011) see http://www. santafe.edu/search/results/?query=Helbingcategory= workingpapers

8. D. Helbing, Human Behaviour and Traffic Networks (Springer, Berlin, 2004)

9. http://www3. wef orum.org/docs/WEF_ITTC_PersonalDataNewAsset_Report_2011.pdf

10. D. Helbing, S. Balietti, EPJ Special Topics 195, 3 (2011) http://arxiv.org/abs/ 1012.0178

11. The 70 online databases that define our planet, Technology Review 12/03/2010, accessible at http://www.technologyreview.com/blog/arxiv/26097/

12. http://www.futurict.eu/the-project/whos-involved 